\title{
Cáncer tiroideo papilar. Reporte de 4 casos familiares
}

\author{
CLARITA FERRADA C. ${ }^{1}$, CLAUDIA GODOY C. ${ }^{2}$, \\ ALEJANDRO MARTÍNEZ A. ${ }^{2}$, HERNÁN GARCÍA B. ${ }^{2}$ \\ 1. Pediatra, becada de Endocrinología infantil, Pontificia Universidad Católica de Chile. \\ 2. Endocrinólogo infantil, Departamento de Pediatría, Hospital Clínico Pontificia Universidad Católica de Chile.
}

\begin{abstract}
Papillary thyroid cancer: case reports of four family cases

Introduction: Papillary thyroid cancer (PTC) is the most common endocrine malignancy, representing $80 \%$ of all thyroid cancers; only $10 \%$ of cases are manifested before age 21 and have an estimated incidence of 0.54 cases per 100,000 people. In children it is diagnosed at a more advanced stage of the disease but with good therapeutic response and very low mortality. Objective: To present four family cases with PTC, discuss the particular characteristics and the importance of early diagnosis. Case reports: 4 families with members affected by family papillary thyroid carcinoma are presented, all pediatric cases were manifested after a direct member adult case was diagnosed, therefore pediatric patients were early detected, but despite a timely treatment, the disease was advanced at the time of diagnosis. The pediatric cases are 3 females and 1 male with an average age of $12 \frac{1}{2}$ years old at diagnosis. Discussion: The family variety of papillary thyroid carcinoma ( 2 or more direct members affected), represents $5 \%$ of papillary cancers. It is transmitted through autosomal dominant inheritance with incomplete penetrance and variable expressivity. It occurs at a younger age than the sporadic type, and it is more aggressive with greater local invasion (32\%), recurrence (20-50\%) and lymphatic metastases (57\%), and it is associated with benign thyroid diseases and often, it is multifocal. Conclusion: The family papillary thyroid cancer is a disease with worse prognosis than the sporadic variety; therefore, a high index of suspicion is required in affected families for early diagnosis and treatment.
\end{abstract}

(Key words: Papillary thyroid cancer, family thyroid cancer, thyroid cancer).

Rev Chil Pediatr 2014; 85 (3): 351-358

\section{RESUMEN}

Introducción: El cáncer papilar del tiroides (CPT) es la neoplasia endocrina más frecuente, siendo el $80 \%$ de los casos de la variedad papilar; sólo el 10\% se manifiesta antes de los 21 años y tiene una incidencia estimada en este grupo de 0,54:100.000. Su comportamiento en la edad pediátrica se caracteriza por el diagnóstico en una etapa más avanzada de la enfermedad pero con buena respuesta terapéutica y muy baja mortalidad.

Recibido el 17 de mayo de 2013, devuelto para corregir el 6 de septiembre de 2013, segunda versión 5 de diciembre de 2013, aceptado para publicación el 9 de diciembre de 2013.

Este trabajo cumple con los requisitos sobre consentimiento /asentimiento informado, comité de ética, financiamiento, estudios animales y sobre la ausencia de conflictos de intereses según corresponda.

Correspondencia a:

Dr. Hernán García Bruce

E-mail: hgarciab@gmail.com 
Objetivo: Presentar 4 casos familiares de CPT, discutir las características particulares y la importancia del diagnóstico precoz en pacientes. Casos clínicos: Se presentan 4 familias con sujetos portadores de un carcinoma papilar familiar de Tiroides, en todas ellas el caso pediátrico se presentó con posterioridad a un caso de un adulto familiar directo, por lo que su búsqueda fue más precoz, y a pesar de un tratamiento oportuno ya tenían enfermedad avanzada al diagnóstico. Los casos pediátricos corresponden a 3 mujeres y 1 varón de edades promedio de 12 años 6 meses al momento del diagnóstico. Discusión: La variedad familiar del carcinoma papilar de tiroides ( 2 o más familiares de primer grado afectados), representa el $5 \%$ de los cánceres papilares. Se transmite a través de herencia autosómica dominante con penetrancia incompleta y expresividad variable. Se manifiesta a menor edad que el esporádico, es más agresivo con mayor invasión local (32\%), recurrencia $(20-50 \%)$ y metástasis linfática $(57 \%)$, y se asocia a enfermedades tiroídeas benignas. Con frecuencia es multifocal. Conclusión: El cáncer familiar papilar de tiroides es una patología con peor pronóstico que la variedad esporádica por lo que se requiere una alto índice de sospecha en las familias afectadas para un diagnóstico y tratamiento precoz.

(Palabras clave: Cáncer papilar de Tiroides, Cáncer Tiroides familiar, cáncer de Tiroides).

Rev Chil Pediatr 2014; 85 (3): 351-358

\section{Introducción}

El cáncer de tiroides constituye la neoplasia endocrina más frecuente dando cuenta del $95 \%$ del total de los cánceres endocrinos infantiles, siendo el carcinoma papilar de tiroides (CPT) responsable del 75-80\% de estos ${ }^{1}$. Sólo un $10 \%$ de los casos ocurre en menores de 21 años con una incidencia estimada en pediatría de 0,54 casos por 100.000 habitantes que corresponden al $1 \%$ de los tumores malignos en menores de 18 años. Se estima que el 3-6\% de los CPT son de origen familiar ${ }^{2}$.

El comportamiento biológico del CPT en pediatría difiere del adulto; se caracteriza por ser diagnosticado en una etapa más avanzada de la enfermedad, a pesar de lo cual muestra buena respuesta al tratamiento con tiroidectomía seguida de terapia con I $131^{1}$ y muy baja mortalidad, presentando una sobrevida del $95 \%$ a 20 años plazo. Estos pacientes tienen mayor frecuencia de persistencia o recurrencia tumoral, aunque presentan una tasa de progresión libre de enfermedad de $65-70 \%$ a 5 años plazo $^{3}$.

Existe una variedad familiar de CPT, con algunas características propias, el carcinoma papilar familiar de tiroides (CPFT) que se define desde el punto de vista clínico como la presencia de CPT en dos o más familiares de primer o segundo grado excluyendo casos que forman parte de algún síndrome genético familiar como Poliposis adenomatosa familiar, Sín- drome de Cowden, Complejo de Carney tipo 1, Síndrome de Werner, Síndrome de Gardner, Síndrome de Peutz-Jeghers o Neoplasia endrocrina múltiple tipo $2^{4}$, o que provengan de zonas expuestas a radiación o deficiencia de yodo ${ }^{5}$. El objetivo es presentar 4 casos familiares de CPT, discutir las características particulares y la importancia del diagnóstico precoz en estos pacientes.

\section{Casos clínicos}

Se presentan 4 familias afectadas por CPFT diagnosticadas en nuestra unidad entre septiembre de 2007 y febrero de 2012 y se describen sus características clínicas e histopatológicas (tabla 1); a los cuatro pacientes se les realizó tiroidectomía total con disección ganglionar central (grupo VI) y luego de más de 25 días de la cirugía se les aplicó Radio Iodo en dosis de 100-150 uCurie con exploración sistémica terapéutica. Los pacientes se mantuvieron con TSH suprimida y fueron controlados periódicamente con Ecografía cervical, T4libre, TSH y tiroglobulina.

\section{Caso 1}

Paciente de sexo femenino 11 años 4 meses de edad, con diagnóstico de tiroiditis autoinmune a los 4 años pesquisada por bocio difuso y antecedente familiar de carcinoma papilar en primo, abuela y bisabuela (figura 1). 
Tabla 1. Resumen casos clínicos

\begin{tabular}{|ccccccccccc|}
\hline Caso & $\begin{array}{c}\text { Edad al } \\
\text { diagnóstico } \\
\text { Años }\end{array}$ & Género & CPFT & $\begin{array}{c}\text { Tiroiditis } \\
\text { crónica }\end{array}$ & & Histología & $\begin{array}{c}\text { Multi- } \\
\text { focal }\end{array}$ & Invasión & Recurrencia & Seguimiento \\
\hline 1 & 9,5 & $F$ & $\begin{array}{c}\text { Primo, tía, } \\
\text { bisabuelo }\end{array}$ & Sí & Papilar & No & No & No & 16 meses \\
\hline 2 & 13 & $F$ & Madre & No & $\begin{array}{c}\text { Variante } \\
\text { esclerosante } \\
\text { difusa }\end{array}$ & Sí & $\begin{array}{l}\text { Local, } \\
\text { vascular, } \\
\text { linfática }\end{array}$ & Sí & 15 meses \\
3 & 13,1 & F & 2 primas & Sí & Papilar & Sí & Local & No & 48 meses \\
4 & 14,5 & M & $\begin{array}{c}\text { Padre, tía } \\
\text { paterna }\end{array}$ & No & Papilar & No & Linfática & No & 24 meses \\
\hline
\end{tabular}

A los 10 años en control ecográfico se pesquisó un nódulo tiroideo izquierdo de 1,3 por $0,9 \mathrm{~cm}$, de bordes mal limitados, heterogéneo, con calcificaciones múltiples en su interior (figura 2), se realizó biopsia por punción con aguja fina cuya histología fue compatible con carcinoma papilar, por lo que se realizó una tiroidectomia total con vaciamiento ganglionar cervical anterior. La biopsia confirmó un Carcinoma papilar de tiroides unifocal, variedad usual, sin infiltración del tejido peritiroideo, sin permeaciones linfovasculares ni compromiso de los linfonodos evaluados, bordes quirúrgicos negativos y resto del tejido tiroideo compatible con una tiroiditis crónica difusa. De acuerdo a protocolo a continuación de la tiroidectomía se aplicó Radio yodo $100 \mathrm{mCi}$ a los 25 días post cirugía con rastreo terapéutico posterior negativo. Permanece sin evidencias de recidiva, con Tiroglobulinas $<0,2 \mathrm{ng} / \mathrm{ml}$ en controles posteriores con 1 año 4 meses de seguimiento.

\section{Caso 2}

Paciente de sexo femenino 14 años 3 meses de edad, con antecedente familiar de madre con carcinoma papilar de tiroides operado. A los 13 años se realizó ecografía tiroídea por bocio que describe lesión sugerente de neoplasia tiroídea extensa con compromiso de mayor parte de la glándula, nódulo dominante lóbulo izquierdo y adenopatías supraclaviculares izquierdas. Teniendo en cuenta sus antecedentes familiares, se decidió realizar una tiroidec-

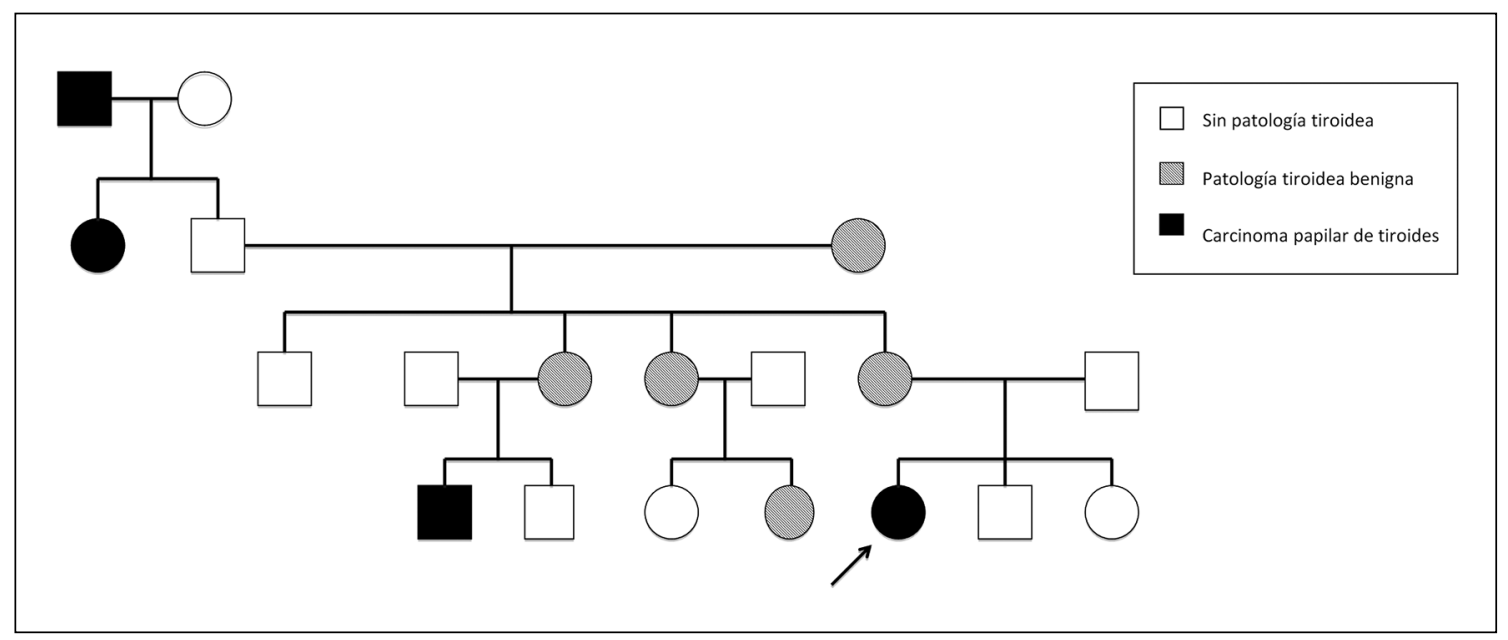

Figura 1. Genograma de la familia de la paciente del caso 1, se señala el caso índice con una flecha. 

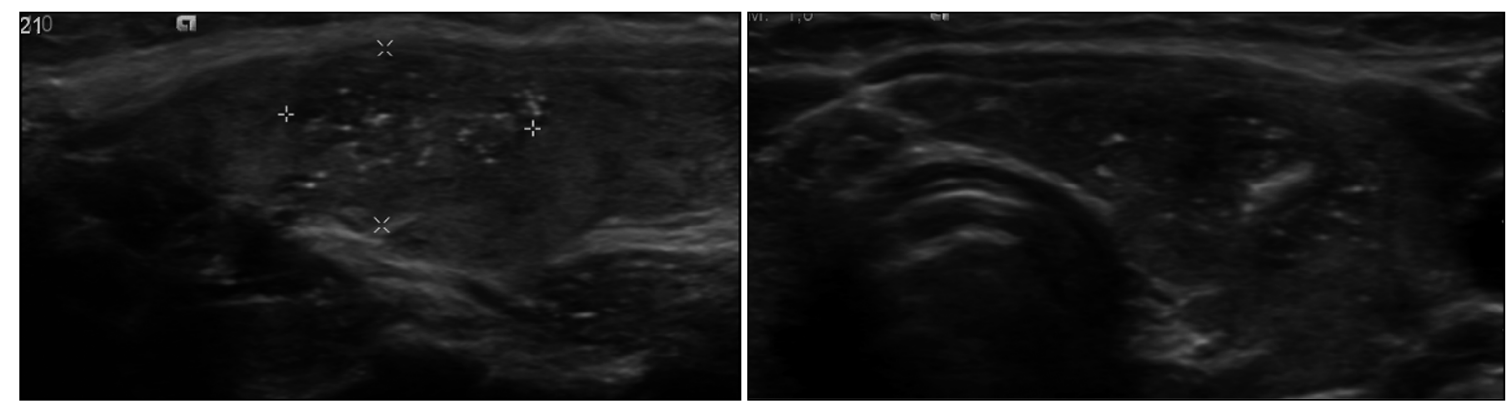

Figura 2. Imágenes ecográficas que permitieron el diagnóstico de la paciente del caso 1, se observan múltiples microcalcificaciones, bordes heterogéneos del nódulo.

tomía total sin punción tiroídea previa, cuya biopsia contemporánea y definitiva confirmaron carcinoma papilar de tiroides en lóbulo izquierdo, itsmo y lóbulo derecho, variante esclerosante difusa que infiltraba el tejido peritiroideo con múltiples permeaciones vasculares y compromiso ganglionar.

A continuación de la Tiroidectomía se trató a los 30 días con radio yodo $150 \mathrm{mCi}$; el rastreo sistémico terapéutico mostró sólo tejido captante en remanente tiroideo. Sin embargo, a los 3 meses post tiroidectomía se pesquisó clínicamente y mediante ecografía, un nódulo en lecho tiroideo derecho con recidiva ganglionar cervical, en la que se confirmó mediante biopsia por punción metástasis de carcinoma papilar en ganglios del grupo IIA, II, IV, VD, por lo que se realizó una reintervención quirúrgica. Evoluciona con persistencia imagenológica de lesión ganglionar cervical sospechosa de recidiva por lo que a los 9 meses postiroidectomia se decidió aplicar una segunda dosis de radio yodo $150 \mathrm{mCi}$ con exploración sistémica terapéutica que mostró aumento de captación en región cervical anterior compatible con la lesión nodular visible ecográficamente. Esta lesión ha disminuido de tamaño en controles posteriores con 1 año 3 meses de seguimiento y persiste con Tiroglobulinas $<0,5 \mathrm{ng} / \mathrm{ml}$.

\section{Caso 3}

Paciente de sexo femenino 18 años 3 meses de edad, diagnóstico de tiroiditis de Hashimoto $\mathrm{y}$ antecedentes familiares de 2 primas hermanas con Carcinoma papilar.
En control ecográfico realizado por los antecedentes familiares a los 13 años de edad se documentó presencia de dos nódulos tiroideos en lóbulo derecho hipoecogénicos y dos en tercio medio y superior de lóbulo izquierdo. Se realizó punción con aguja fina en 2 de los nódulos que resulta sugerente de cáncer papilar de tiroides. Se realizó tiroidectomía total con vaciamiento ganglionar cervical anterior, biopsia confirmó el diagnóstico de carcinoma papilar con compromiso ganglionar. A los 28 días post cirugía se administró radio yodo 150 $\mathrm{mCi}$ con exploración sistémica negativa.

Después de 4 años de seguimiento, las Tiroglobulinas se mantienen frenadas y no hay signos de metástasis ni recaída.

\section{Caso 4}

Paciente de sexo masculino 16 años 3 meses de edad, con antecedentes de carcinoma papilar de tiroides del padre y una tía paterna; por esta razón a los 14 años se realizó un tamizaje con ecografía tiroídea que mostró nódulo paraitsmico a derecha de aspecto sólido, con microcalcificaciones. Punción con aguja fina reveló hallazgos morfológicos compatibles con carcinoma papilar; por lo que se realizó tiroidectomía total, cuya biopsia confirmó diagnóstico de carcinoma papilar multifocal variedad usual con metástasis linfáticas, sin compromiso de bordes quirúrgicos. A los 40 días post cirugía se administra radio yodo $150 \mathrm{mCi}$. La exploración sistémica posterior fue compatible con remanente tiroideo. Permanece con Tiroglubulinas frenadas sin signos de recidiva a los 2 años de evolución post cirugía. 


\section{Discusión}

Hemos descrito 4 casos de Cáncer Papilar de Tiroides en niños, con antecedentes de al menos 1 adulto afectado en su familia directa, lo que es la base para plantear el diagnóstico de CPFT, entidad reconocida previamente descrita en la literatura. Su epidemiología se basa hasta el momento en estudios descriptivos de casos y controles, los que han reportado un riesgo de desarrollar un carcinoma de tiroides en familiares de primer grado de pacientes con cáncer papilar 5 a 10 veces mayor que en la población general ${ }^{6}$.

Se estima que el CPFT representa el 3 al $9 \%$ de todos los casos de cáncer de tiroides ${ }^{7}$, aunque su incidencia real es desconocida, ya que se ha descrito en series de casos catalogados como CPFT por 2 familiares afectados una tasa del 45 al $69 \%$ de casos esporádicos, en estas mismas series de casos se ha observado que los casos catalogados como CPFT con 3 o más familiares afectados con Ca papilar, la probabilidad de que sea familiar y no esporádico aumenta en un $96 \% 5$.

El CPFT al igual que la variedad esporádica, afecta más a las mujeres que a hombres en proporción de $3: 1$, pero la edad al diagnóstico es menor (promedio: 39-43 vs 46-49 años) ${ }^{5} \mathrm{y}$ se ha demostrado una "anticipación clínica", es decir, la segunda generación de afectados debuta a menor edad y con enfermedad más avanzada $^{8}$.

La patogenia y las bases genéticas del CPFT no están bien caracterizadas. Se postula que presenta un patrón de herencia autosómica dominante con penetrancia incompleta y expresividad variable, y/o una herencia poligénica.

No se ha identificado aún un gen responsable del CPFT; Se han identificado 6 regiones como candidatos potenciales de susceptibilidad sin la participación de los genes ya descritos en otros tumores tiroideos como los genes RET, TRK, MET, APC, PTEN ni TSHR (tabla 2) ${ }^{9}$.

El CFPT se presenta clínicamente en forma similar al Cáncer Papilar esporádico, pero es más grave; se asocia habitualmente a enfermedades tiroídeas benignas como adenomas foliculares, tiroiditis de Hashimoto, bocio multinodular, hipotiroidismo o hipertiroidismo en el $45 \%$ (36-57\%) de los casos ${ }^{10}$. En estos pacientes existe mayor riesgo de presentar otras neoplasias principalmente cáncer de mama, riñón, colon, vesícula, melanomas y linfomas ${ }^{10}$.

Con respecto al pronóstico, existe evidencia que sugiere que el CPFT se comporta en forma más agresiva que la variedad esporádica con mayor invasión local, multifocalidad, extensión extratiroidea y metástasis linfáticas al debut ${ }^{11,12}$; además de mayor riesgo de recurrencia siendo el antecedente de historia familiar un factor de riesgo independiente para esta complicación $^{2,13}$ (tabla 3), aunque es un tema aún en discusión debido a que hay estudios que contradicen estos hallazgos ${ }^{5}$.

Comparando sujetos afectados en la $2 \mathrm{da}$ generación con CPFT estos debutarían a menor edad (promedio: 39-43 vs 46-49 años), tendría mayor invasión extratiroidea (58 vs 29\%) y mayor tasa de recurrencia (50 vs 19\%) ${ }^{12}$.

Tabla 2. Regiones potenciales de susceptibilidad para carcinoma papilar familiar de tiroides

\begin{tabular}{|ll|}
\hline Región potencial de susceptibilidad & Características clínicas \\
MNG1 (14q32) & Familia con 18 casos de bocio multinodular y 2 casos de carcinoma papilar \\
$19 p 13.2$ & $\begin{array}{l}\text { Carcinoma tiroideo con oxifilia, } 3 \text { generaciones de una familia con } 6 \text { miembros con } \\
\text { bocio multinodular y } 3 \text { con PTC }\end{array}$ \\
$1 q 21$ & Sería un gen de susceptibilidad en que PTC se asocia a neoplasia papilar renal \\
\hline $2 q 21$ & \\
$8 p 23.1-p 22$ & \\
Complejo telomerasa-telomerasa & \\
\hline
\end{tabular}


Tabla 3. Comparación CTNM familiar vs esporádico

\begin{tabular}{|lcc|}
\hline Característica & Carcinoma esporádico & Variedad familiar \\
\hline Edad de presentación & 50 años & 10 años menos (40 años) \\
Bilateral & $19 \%$ & $43 \%$ \\
Multifocal & $20-30 \%$ & $42-93 \%$ \\
Invasión local & $4-16 \%$ & $32 \%$ \\
Recurrencia & $5-15 \%$ & $20-50 \%$ \\
Hiperplasia multinodular & $29 \%$ & $41 \%$ \\
MT linfáticas & $38-43 \%$ & $57 \%$ \\
Mutación BRAF & $40 \%$ & $0 \%$ \\
\hline
\end{tabular}

Referencia: Sippel R, Caron N, Clark O. An Evidence-based Approach to Familial Nonmedullary Thyroid Cancer: Screening, Clinical Management, and Follow-uP. World J Surg 2007; 31: 924-33.

Tabla 4. Recomendaciones para el diagnóstico precoz CPFT ${ }^{8}$

\begin{tabular}{|ll|}
\hline Criterio de riesgo & Recomendación \\
\hline 1 familiar de primer grado afectado & Evaluación con historia y examen físico \\
\hline Diagnóstico de bocio adenomatoso & Screening familiar incluyendo ecografía aunque no existan antecedentes familiares de CPFT \\
2 o más familiares afectados & Historia, examen físico, ecografía tiroídea, seguimiento anual \\
\hline
\end{tabular}

Se recomienda comenzar el screening 10 años antes de la edad del pariente diagnosticado más joven o máximo a los 20 años de edad. Referencia: Nosé V. Familial Non-Medullary Thyroid Carcinoma: An Update. Endocr Pathol 2008; 19: 226-40.

Considerando el debut más precoz y la evolución más agresiva de estos casos, debería realizarse tamizaje en todos los miembros mayores de cierta edad, de una familia con 2 o más personas con diagnóstico de CPFT, aunque estos sean asintomáticos. El screening debe incluir detallada historia familiar, antecedentes de exposición ambiental, presencia de otras patologías que puedan formar parte de algún síndrome genético que se asocie a CPT; se debe buscar síntomas locoregionales como dolor, disfagia, disnea y masas cervicales, ya que ha sido demostrado que la invasión local y la enfermedad extratiroidea están aumentadas en estos pacientes. Debido al mayor riesgo de enfermedad tiroídea benigna y el comportamiento más agresivo de la variedad CPFT se debe además como parte del estudio realizar una ecografía tiroídea que permitirá la detección de nódulos tiroideos no palpables, cáncer tiroideo oculto y evaluación de compromiso extratiroideo $^{5}$. Se ha descrito hallazgo de nódulos por ecografía en el $52 \%$ de los familiares, de los cuales $10 \%$ eran carcinomas papilares ${ }^{10}$.
Con respecto al estudio de nódulos tiroideos en estos pacientes con punción con aguja fina, esta técnica tendría menor rendimiento como test diagnóstico debido a la alta incidencia de patología benigna en estos pacientes.

No hay consenso sobre la edad precisa en que debe comenzar a realizarse el screening familiar. Se ha observado que los casos de CPFT debutan 10 años antes que los casos esporádicos, por lo que es razonable comenzar el screening a los 10 años de edad o al menos 10 antes de la edad al diagnóstico del familiar de menor edad con CPFT (tabla 4$)^{5}$.

Nuestros casos son ilustradores de las características más agresivas del CPFT con respecto al cáncer esporádico de tiroides. Las recomendaciones de manejo reúnen una evidencia limitada, tipo $\mathrm{C}$, es decir estudios de casos y controles, retrospectivos y opinión de expertos, no existiendo aún guías clínicas para esta patología.

Existe acuerdo en recomendar en todos los pacientes con CPTF tiroidectomía total; junto a ello debe ser considerada la disección gan- 
glionar central (grupo VI) de rutina en caso de tumores mayores a $1 \mathrm{~cm}$, con sospecha ecográfica de compromiso ganglionar, comportamiento agresivo de la enfermedad en otros miembros de la familia (extensión extratiroidea, multifocalidad, compromiso ganglionar al diagnóstico) o presencia de 3 o más miembros de su familia afectados.

No existe evidencia suficiente para hacer recomendaciones especificas sobre el tratamiento con radio $\mathrm{I}$, pero de acuerdo a las características de este tumor lo recomendable sería aplicarlo en todos los casos, y mantener suprimida la TSH por un período más prolongado, más aun en los casos con comportamiento más agresivo de la enfermedad o aquellos con antecedente de más de 3 familiares afectados.

Se presentan 4 familias portadoras de Carcinoma Papilar familiar de Tiroides, 2 de los cuales tenían antecedente de patología tiroídea benigna como está descrito en la literatura; la mayor parte de ellos ya tenían invasión local y linfática al diagnóstico a pesar de que este se hizo en forma precoz entre los 9 y 15 años de edad, confirmando la mayor agresividad del CPFT. Todos los pacientes fueron manejados con tiroidectomía total, disección ganglionar central y Radio Iodo después de 25 días postcirugía con dosis entre 100 y $150 \mathrm{mCi}$. Un seguimiento de 1-4 años en estos pacientes, ha demostrado niveles indetectables de Tiroglobulina en todos ellos, excepto un paciente que presentó recurrencia y debió ser reintervenido, con buena respuesta a la segunda cirugía. Aunque aún el seguimiento es limitado, existen buenas razones para suponer un buen pronóstico en nuestros casos, demostrando que la intervención precoz basada en un índice de sospecha mayor por la presencia de otros casos en la familia directa, mejora el pronóstico y la evolución del CPFT.

\section{Conclusión}

El cáncer papilar familiar de tiroides es más agresivo y de peor pronóstico que la variedad esporádica por lo que se requiere una alto índice de sospecha en los hijos (hombres y muje- res) y otros familiares directos de los pacientes afectados para un diagnóstico precoz y un tratamiento efectivo.

Debe sospecharse en lesiones bilaterales, multifocales y multinodulares y cuando se diagnostica en niños o pacientes de sexo masculino ${ }^{2}$.

En la actualidad no existe relación causal entre mutación de algún gen específico y CPFT, por lo que el diagnóstico es clínico.

Nuestra recomendación es realizar screening con ecografía a todos los familiares de un paciente con diagnóstico de carcinoma papilar de tiroides a partir de los 10 años o al menos 10 años antes de la edad de diagnóstico del familiar con debut más precoz.

\section{Referencias}

1.- Mosso L: Cáncer Papilar de tiroides a la luz de las evidencias actuales. Revista Chilena de Endocrinología y Diabetes 2008; 1 (3): 182-9.

2.- Lee Y, Yoon J, Yi O, Sung T, Chung K, Kim W: Familial History of Non-Medullary Thyroid Cancer Is an Independent Prognostic Factor for Tumor Recurrence in Younger Patients With Conventional Papillary Thyroid Carcinoma. Journal of Surgical Oncology 2013; Published online in Wiley Online Library (wileyonlinelibrary. com).

3.- Parisi M, Mankoffsemin D: Differentiated Pediatric Thyroid Cancer: Correlates With Adult Disease, Controversies in Treatment. Nucl Med 2007; 37: 340-56.

4.- Nosé $V$ : Familial thyroid cancer: a review. Modern Pathology 2011; 24: S19-S33.

5.- Mazeh H, Sippel R: Familial Nonmedullary Thyroid carcinoma. Thyroid 2013; 23 (9): 1049-56.

6.- Malchoff C, Malchoff D: Familial Nonmedullary Thyroid Carcinoma. Seminars in Surgical Oncology 1999; 16: 16-8.

7.- Vriens M, Suh I, Moses W, Kebebew E: Clinical Features and Genetic Predisposition to Hereditary, Nonmedullary Thyroid Cancer. Thyroid 2009; 19 (12): 1343-9.

8.- Malchoff C, Malchoff D: Familial Nonmedullary Thyroid Carcinoma. Cancer Control 2006; 13 (2): 10610.

9.- Khan A, Smellie J, Nutting C, Harrington K, Newbold $K$ : Familial Nonmedullary Thyroid Cancer a Review of the Genetics. Thyroid 2010; 20 (7): 795-801.

10.- Sippel R, Caron N, Clark O: An Evidence-based Ap- 
proach to Familial Nonmedullary Thyroid Cancer: Screening, Clinical Management, and Follow-uP. World J Surg 2007; 31: 924-33.

11.- Nosé V: Familial Non-Medullary Thyroid Carcinoma: An Update. Endocr Pathol 2008; 19: 226-40.
12.- Park Y, Ahn H, Choi H, Kim K, Park D, Cho B: The Long-Term Outcomes of the Second Generation of Familial Nonmedullary Thyroid Carcinoma Are More Aggressive than Sporadic Cases. Thyroid 2012; 22 (4): 356-62. 